\title{
A Dynamic Approach to Locating Memory Leaks
}

\author{
Kostyantyn Vorobyov $^{1}$, Padmanabhan Krishnan ${ }^{2}$, and Phil Stocks ${ }^{1}$ \\ 1 Centre for Software Assurance, Bond University, Gold Coast, Australia \\ \{kvorobyo,pstocks\}@bond. edu . au \\ ${ }^{2}$ Oracle Labs, Brisbane, Australia \\ paddy.krishnan@oracle.com
}

\begin{abstract}
We present a dynamic approach to memory leak detection and reporting to aid the debugging process. We track memory allocations and aliases during execution, which allows us not only to detect leaks, but also locate the point of the leak in the source code. The level of tracking can be customised by the user. This analysis is safe in the presence of pointer aliasing - a benefit of the dynamic approach, as such problems are hard to solve using static analysis (or lead to many false positives). Our technique works by instrumenting programs with statements that track memory allocations, capture alias information, and monitor potential leaks. By tracking only location and size of memory allocation, rather than monitoring every bit, as is common in other approaches, we keep memory overhead very low. We demonstrate the applicability of our approach on a number of open-source programs and a few SPEC CPU benchmarks. Our experiments show that the overheads compare favourably with current analysis tools such as Valgrind.
\end{abstract}

Keywords: Memory leak, Program instrumentation, Monitoring.

\section{Introduction}

Memory monitoring through program instrumentation has been extensively researched for the past two decades, resulting in a number of proprietary and open source tools, such as Purify [1], Valgrind [2], Insure++ [3], Intel Parallel Inspector 4 and others. These tools are often used during test phase, as dynamic analysis is reliable and safe in the presence of pointer aliasing - a problem, for which static techniques do not scale or lead to many false positives. For example, it has become a common practice to monitor test-suite execution, which allows to automatically enable additional oracles for the cost of extra overhead.

The state-of-the-art monitoring techniques for memory leak detection use binary instrumentation, which injects code that observes execution and detects memory defects at the assembly level. This allows the monitoring of each operation, including operations that occur in third party libraries. Even though proven to be useful, binary instrumentation techniques have a number of limitations. Firstly, as it observes every operation and tracks each bit of allocated memory, binary instrumentation is known to produce massive memory and run-time

H. Yenigün, C. Yilmaz, and A. Ulrich (Eds.): ICTSS 2013, LNCS 8254, pp. 255-270, 2013.

(C) IFIP International Federation for Information Processing 2013 
overheads. While this may have little impact during the development process, it makes it hard to deploy these tools in performance testing, where runs of unmodified programs may take a long time to execute. Additionally, these tools default to reporting memory leaks using locations of allocation, which does not always provide enough information to eliminate detected defects. Finally, as instrumentation is performed at the assembly level, binary instrumentation techniques are inevitably platform and architecture dependent.

In this paper we present a monitoring approach to memory leak detection that reports where the leakage occurs. This information can facilitate the debugging process. In contrast to binary instrumentation we modify source code of programs by inserting statements to record and update memory state, observe execution and detect memory leaks. In our approach we track memory at the block level, recording only locations and sizes of allocated blocks, which avoids monitoring of every bit and minimises memory overheads. We associate each tracked block with two types of locations: allocation and access. The allocation locations are assigned only once when blocks are created on the heap. The locations of access are updated based on the execution of the program. Every time a block containing references is updated, the access locations are also updated to reflect reachability of the block via some program variable. This is achieved by dynamically computing the dereference of a block's address space. The dereference computation is tunable by abstractly specifying memory areas that do not contain pointers and therefore can not leak. This yields a technique where run-time overheads can be reduced for the cost of reporting less debugging information without losing precision. At the end of execution we report unreachable blocks that have not been de-allocated along with the information where it was allocated and where the leakage occurred.

We have a prototype implementation (called Skiff) for monitoring C programs. We demonstrate the applicability of our approach by analysing real UNIX programs and SPEC benchmarks and report the results of our experimentation.

The overall contributions made by this paper are as follows:

- A tunable monitoring approach to memory leak detection that uses source code instrumentation and identifies locations of leakage.

- A proof-of-concept implementation of our technique.

- An empirical evaluation of our approach by comparing the results produced by our tool to the results produced by a state-of-the-art memory profiler. This evaluation demonstrates that in observing test suites of applications the overheads introduced by our technique are much lower.

The rest of the paper is organised as follows. In Section 2 we discuss syntax and semantics of a simple imperative language we use to describe our technique at the abstract level. Section 3 presents a technical description of our approach and shows how to apply it on $\mathrm{C}$ programs. Section 4 discusses empirical results of a prototype implementation and Section 5 gives an overview of related work. We give our concluding remarks and discuss future directions in Section 6 . 


\section{Syntax and Memory Semantics}

We present our approach at the abstract level using a simple imperative language. We now describe the syntax and semantics of this language.

Figure 1 shows an abstract imperative language similar to the WHILE [5] programming language extended with memory allocation and operations for manipulating pointers.

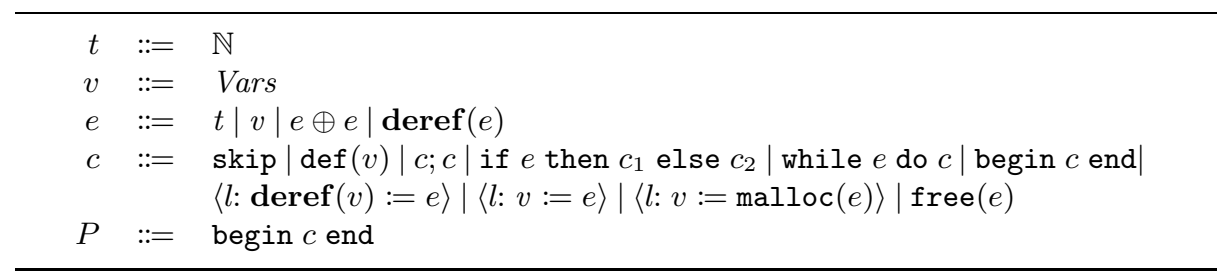

Fig. 1. Abstract Language

Expressions $e$ consist of constants $t \in \mathbb{N}$, variables $v \in$ Vars (where set Vars denotes the set of variables), composite expressions $e \oplus e$, where $\oplus$ is a binary operator and dereference operator $\operatorname{deref}(e)$. Command $c$ consists of atomic commands (skip), variable definitions $(\operatorname{def}(v)$ ), conditional expressions (if $e$ then $c_{1}$ else $c_{2}$ ), loops (while $e$ do $c$ ), scopes (begin $c$ end), sequential composition $(c ; c)$, labelled assignments $\langle l: v:=e\rangle$ and $\langle l: \operatorname{deref}(v):=e\rangle$, where label $l$ identifies source location of the command (e.g., a source code line number) and built-in memory allocation and de-allocation commands $\langle l: v=\operatorname{malloc}(e)\rangle$ and $\mathrm{free}(e)$ respectively. The command malloc allocates a new memory block (e.g., a sequence of contiguous memory cells) of size specified by expression $e$ and binds the address of the first cell in the allocated segment to a variable $v$. free $(e)$ de-allocates a memory block, whose first address is given by expression $e$. If no such block exists the statement is equivalent to skip. Program $P$ is a sequence of commands within a scope.

We now introduce our semantics of the memory model to formally define memory leaks. We let Scope denote the set of scope identifiers and set $\mathbb{N}$ to represent a memory address. A particular memory block is denoted by a pair over $\mathbb{N}$ representing start and end addresses of the block. Let $\mathcal{B}=\mathbb{N} \times \mathbb{N}$ be the set of all blocks. Then, memory allocation is a subset of such pairs. Formally, the set of all possible allocations $\mathcal{A}$ is $\mathcal{P}(\mathcal{B})$, where $\mathcal{P}$ is the powerset operator. Typical elements are denoted by $\sigma$ and we require that allocated blocks are disjoint, and that the start address is not greater than the end address (and assume blocks represent allocation of contiguous chunks of memory).

Memory mapping $m_{\sigma}$ is the set of pairs $\mathbb{N} \times \mathbb{N}$, where each pair $(i, t) \in m_{\sigma}$ represents a valid memory block. The set of all possible memory mappings is denoted by the set $\mathcal{M}=\mathcal{P}(\mathbb{N} \times \mathbb{N})$, that is $m_{\sigma}$ is an element of $\mathcal{M}$. A memory mapping is valid only if each address mapped to a value lies within the allocated block. 
Store usage by program (denoted $\mu$ ) is the set of triples Vars $\times$ Scope $\times \mathbb{N}$, where a triple $(v, s, t) \in \mu, v \in$ Vars, $s \in$ Scope, $t \in \mathbb{N}$ in a particular state represents a variable $v$ defined at scope $s$ and bound to a numeric constant $t$. Formally, the set of all possible store usages $\mathcal{S}$ is $\mathcal{P}($ Vars $\times$ Scope $\times \mathbb{N})$, where store usage in a particular state $\mu$ is an element of $\mathcal{S}$.

Finally, we let the set $L a b$ denote the set of all program labels. An element $l \in L a b$ denotes either a defined source location (such as a line number) or an undefined one (denoted by $\perp$ ). We use labels to track usage of blocks during memory allocation and assignments. We let function loc $: \mathbb{N} \times \mathbb{N} \rightarrow$ Lab denote usage tracking in a particular state. For example, a label associated with a block $(a, b) \in \sigma$, where $\sigma$ is a valid allocation, is retrieved using $\operatorname{loc}(a, b)$. The set of all such functions is denoted $L t$ (for label tracking).

\subsection{Operational Semantics}

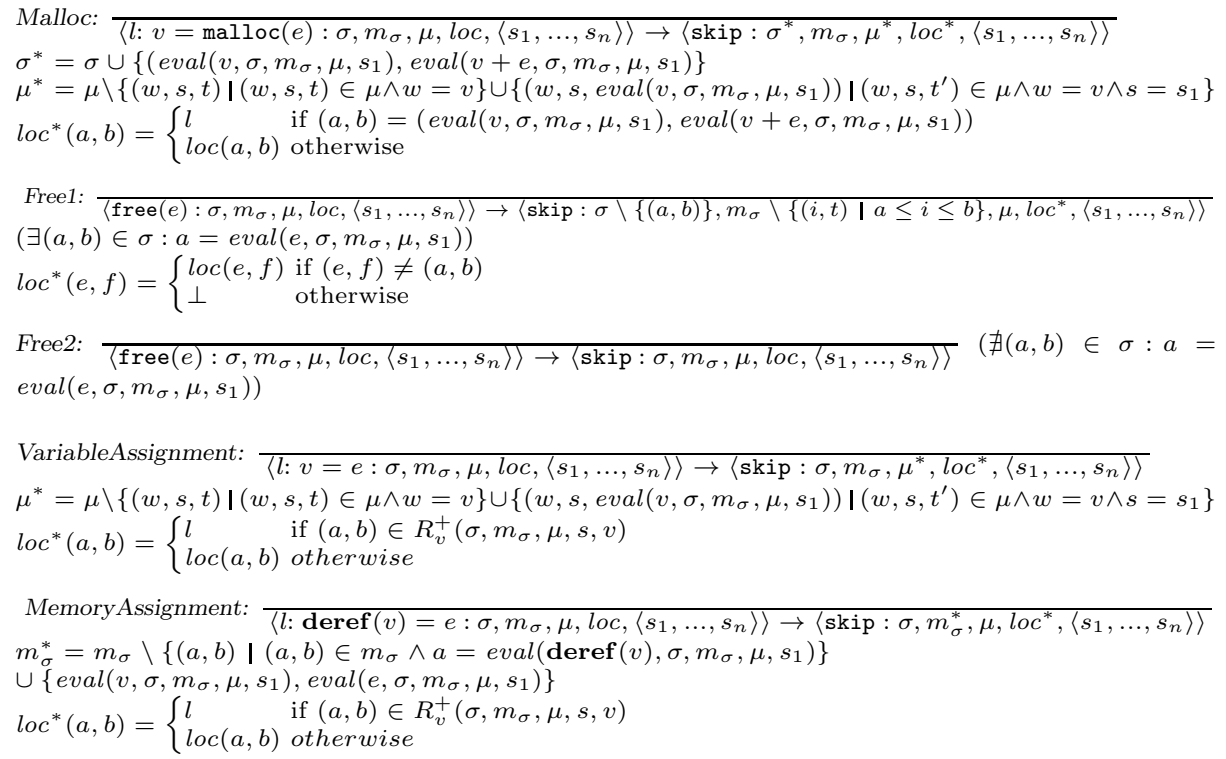

Fig. 2. Operational Semantics

The operational semantics of commands (with key rules shown in Figure 2) is defined as a relation $\rightarrow$ on configurations, where a configuration is a tuple $\left\langle c: \sigma, m_{\sigma}, \mu\right.$, loc,$\left.S c o p e^{*}\right\rangle$, such that $c$ is a program command, $\sigma$ is a memory allocation, $m_{\sigma}$ is a memory mapping, $\mu$ is a store usage, loc is a function that identifies command labels associated with allocated memory blocks, and Scope* is a sequence of scope identifiers (e.g., sequence $\left\langle s_{1}, \ldots, s_{n}\right\rangle$, where $s_{1}, \ldots, s_{n} \in$ Scope are distinct identifiers of executed scopes). 
The rule for $\langle l: v:=\operatorname{malloc}(e)\rangle$ adds a new block to $\sigma$ and updates the block pointed to by $v$ to the new block (indicated by the change to $\mu$ ). The rule for free(e) removes the block identified by $e$ from $\sigma$. Rule $\langle l: v:=e\rangle$ for variable assignments update store usage, while rule $\langle l$ : $\operatorname{deref}(v):=e\rangle$ for memory assignments modifies memory mapping, i.e., allows to write to a memory location through a variable dereference. Additionally, each of the above rules modify label function loc, which associates labels with allocated blocks and thus is the key to label tracking. We discuss this behaviour in detail in Section 3 . The rules for the other commands are standard and are not presented.

In the next few paragraphs we summarise the key concepts underlying our technique. We elaborate only on concepts that are relevant for memory leaks. The behaviour of expressions is standard in that the result of their evaluation is defined by function $\operatorname{eval}\left(e, \sigma, m_{\sigma}, \mu, s\right)$ that evaluates to a constant $t \in \mathbb{N}$, where $e$ is an expression, $\sigma$ is a memory allocation, $m_{\sigma}$ is a memory mapping, $\mu$ is a store usage and $s \in S c o p e$ is an executed scope. I.e., $\operatorname{eval}\left(e, \sigma, m_{\sigma}, \mu, s\right)=t$ denotes expression $e$ that evaluates to a constant $t$ in scope $s$, where $\sigma \in \mathcal{A}$ is a valid allocation, $m_{\sigma} \in \mathcal{M}$ is a valid memory mapping and $\mu \in \mathcal{S}$ is a store usage.

A variable $v$ in scope $s$ points to a memory block if the value in the variable lies within that block. This is formally given using Definition 1

Definition 1 (Points to via variable). Given a valid memory allocation $\sigma \in$ $\mathcal{A}$, valid memory mapping $m_{\sigma} \in \mathcal{M}$, store usage by program $\mu \in \mathcal{S}$, scope $s \in$ Scope and variable $v \in$ Vars, $v$ is said to point to an allocated memory block $(a, b) \in \sigma$ in scope $s$ if and only if $a \leq \operatorname{eval}\left(v, \sigma, m_{\sigma}, \mu, s\right) \leq b$.

Given a valid memory allocation $\sigma$, valid memory mapping $m_{\sigma}$, store usage $\mu$ and scope $s$, a given block $(a, b) \in \sigma$ can point to another block $(c, d) \in \sigma$ in scope $s$, if and only if a memory address within $(a, b)$ is mapped to an address that lies within $(c, d)$. The relation $R_{b}$ in Definition 2 defines this formally.

Definition 2 (Points to via block). Given a valid memory allocation $\sigma \in \mathcal{A}$, valid memory mapping $m_{\sigma} \in \mathcal{M}$, store usage by program $\mu \in \mathcal{S}$, scope $s \in$ Scope and allocated memory block $(a, b) \in \sigma$, binary relation:

$$
\begin{aligned}
& R_{b}\left(\sigma, m_{\sigma}, \mu, s,(a, b)\right)=\{(e, f) \mid(e, f) \in \sigma \wedge \\
& \left.\quad \exists i \in \mathbb{N}: a \leq i \leq b \wedge e \leq \operatorname{eval}\left(\operatorname{deref}(i), \sigma, m_{\sigma}, \mu, s\right) \leq f\right\}
\end{aligned}
$$

defines the set of memory blocks in $\sigma$, block $(a, b)$ points to.

Thus, in allocation $\sigma$, memory mapping $m_{\sigma}$, store usage $\mu$ and scope $s$, block $(a, b)$ points to block $(c, d)$ if and only if $(c, d) \in R_{b}\left(\sigma, m_{\sigma}, \mu, s,(a, b)\right)$.

In a memory allocation $\sigma$, memory mapping $m_{\sigma}$, store usage $\mu$ and scope $s$, a given block (say $b_{n}$ ) is accessible from another block (say $b_{0}$ ) if there is a sequence of blocks $b_{1}, \cdots, b_{n-1}$ such that for all $i$ between 0 and $n-1, b_{i}$ points to $b_{i+1}$. This is formally defined by the relation $R_{b}^{+}$in Definition 3 . 
Definition 3 (Accessibility). Given a valid memory allocation $\sigma \in \mathcal{A}$, valid memory mapping $m_{\sigma} \in \mathcal{M}$, store usage by program $\mu \in \mathcal{S}$, scope $s \in$ Scope and allocated memory block $(a, b) \in \sigma$, binary relation:

$$
\begin{aligned}
R_{b}^{+}\left(\sigma, m_{\sigma}, \mu, s,(a, b)\right) & =\left\{(e, f) \mathbf{I}(e, f) \in R_{b}\left(\sigma, m_{\sigma}, \mu, s,(a, b)\right) \vee\right. \\
& \left(\exists(c, d) \in R_{b}\left(\sigma, m_{\sigma}, \mu, s,(a, b)\right):(e, f) \in R_{b}^{+}\left(\sigma, m_{\sigma}, \mu, s,(c, d)\right)\right\}
\end{aligned}
$$

defines the set of blocks accessible from $(a, b)$.

Thus, for a memory allocation $\sigma$, memory mapping $m_{\sigma}$, store usage $\mu$ and scope $s$, memory block $(c, d) \in \sigma$ is accessible from block $(a, b) \in \sigma$ if and only if $(c, d) \in R_{b}^{+}\left(\sigma, m_{\sigma}, \mu, s,(a, b)\right)$.

Given a memory allocation $\sigma$, block $(a, b) \in \sigma$, memory mapping $m_{\sigma}$, store usage $\mu$ and scope $s$, variable $v \in \operatorname{Vars}$ references $(a, b)$ if $v$ points to $(a, b)$ or there exists some block $(c, d) \in \sigma$, such that $v$ points to $(c, d)$ and $(a, b)$ is accessible via $(c, d)$. This is formally defined by the relation $R_{v}^{+}$in Definition 4

Definition 4 (Reference). Given a valid memory allocation $\sigma \in \mathcal{A}$, block $(a, b) \in \sigma$, valid memory mapping $m_{\sigma} \in \mathcal{M}$, store usage $\mu \in \mathcal{S}$, scope $s \in$ Scope and variable $v \in$ Vars, binary relation

$$
\begin{aligned}
& R_{v}^{+}\left(\sigma, m_{\sigma}, \mu, s, v\right)=\left\{(a, b) \mid \mathrm{I}(a, b) \in \sigma: a \leq \operatorname{eval}\left(v, \sigma, m_{\sigma}, \mu, s\right) \leq b \vee\right. \\
& \left.\quad\left(\exists(c, d) \in \sigma: c \leq \operatorname{eval}\left(v, \sigma, m_{\sigma}, \mu, s\right) \leq d \wedge(a, b) \in R_{b}^{+}\left(\sigma, m_{\sigma}, \mu, s,(c, d)\right)\right)\right\}
\end{aligned}
$$

defines the set of blocks referenced by variable $v$.

Thus, given a memory allocation $\sigma \in \mathcal{A}$, memory mapping $m_{\sigma} \in \mathcal{M}$, store usage by program $\mu \in \mathcal{S}$ and scope $s \in$ Scope, variable $v \in$ Vars references allocated memory block $(a, b) \in \sigma$, if $(a, b) \in R_{v}^{+}\left(\sigma, m_{\sigma}, \mu, s, v\right)$.

Given the above we can define memory leak as follows:

Definition 5 (Memory leak). Given a valid memory allocation $\sigma \in \mathcal{A}$, valid memory mapping $m_{\sigma} \in \mathcal{M}$, store usage $\mu \in \mathcal{S}$ and scope $s$, a memory block $(a, b) \in \sigma$, is a memory leak with respect to store usage by program $\mu$, if there exists no triple $(v, s, t) \in \mu, v \in$ Vars, $s \in$ Scope, $t \in \mathbb{N}$, such that $(a, b) \in$ $R_{v}^{+}\left(\sigma, m_{\sigma}, \mu, s, v\right)$. That is, block $(a, b)$ is not referenced by program variables from the given store usage.

\section{Memory Leak Detection}

We now present technical details of the memory leak detection technique. Our approach consists of two stages: static and dynamic. At the static stage we instrument an input program (say $P$ to obtain $P^{\prime}$ ) with statements that monitor the execution of $P$ to detect memory leaks. At the dynamic stage we run $P^{\prime}$, which reports occurred memory leaks (if any) at the end of its execution.

To keep track of the memory state during the execution of $P$ we use an explicit data-structure $T_{\sigma}$. A particular state of $T_{\sigma}$ describes the state of memory that has been tracked during the execution of the transformed program $P^{\prime} . T_{\sigma}$ is the set of 4 -tuples of values (i.e., $\left.T_{\sigma} \in \mathcal{P}(\mathbb{N} \times \mathbb{N} \times \mathbb{N} \times \mathbb{N})\right)$. An element $\left(a, b, l_{a}, l_{u}\right)$ of $T_{\sigma}$ 
represents a memory block $(a, b)$ (where $a$ and $b$ are its start and end addresses), such that at program location $l_{a}$ block $(a, b)$ was allocated and referenced via a variable at label $l_{u}$. We further refer to labels $l_{a}$ and $l_{u}$ as to allocation and usage labels respectively.

We now describe functions that operate on $T_{\sigma}$. The function $\operatorname{insert}\left(T_{\sigma}, \mathrm{a}, \mathrm{b}, \mathrm{l}\right)$ adds the element $(a, b, l, 0)$ (where 0 represents an undefined label) to $T_{\sigma}$. The function delete $\left(T_{\sigma}, a\right)$ removes elements whose start address is $a$. Given a memory address $c$ the function lookup $\left(T_{\sigma}, c\right)$ searches through the elements of $T_{\sigma}$ and returns a memory block $(a, b)$, if there exists an element $\left(a, b, l_{a}, l_{u}\right)$ of $T_{\sigma}$, such that $c$ lies within $(a, b)$, or a pair $(0,0)$ (where $(0,0)$ represents an invalid block) otherwise. The function update $\left(T_{\sigma}, a, l\right)$ modifies a usage label of a particular element of $T_{\sigma}$ identified by a start address of the tracked memory block it represents.

The function updateLabel is the main memory tracking function. Its task is to update usage labels of memory blocks that participated in assignments. Informally, a usage label associated with a block of memory identifies a source location at which that memory block was accessible. For example, given an assignment statement $\langle l: v=e\rangle$, where $v$ is a variable and $e$ is an expression, updateLabel sets usage labels of all memory blocks referenced by $v$ to $l$.

The semantics of updateLabel is as follows. Given an input value val (such that $v a l$ is a value to which some variable $v$ at label $l$ evaluates), updateLabel identifies the set of memory blocks (say $R$ ) referenced by $v$ (i.e., memory blocks that can be accessed through $v$ ). $R$ is populated by recursively dereferencing values stored in blocks pointed to by $v$ and then calling lookup on the values obtained by dereferencing. Valid memory blocks returned by lookup are then added to $R$. For example, given that $v$ points to a memory block $(a, b)$, which in turn points to some block $(c, d)$, updateLabel first dereferences the value of $v$, identifies $(a, b)$ as being pointed to by $v$ and adds it to $R$. Further, it dereferences each value in the range $[a, b]$ and adds $(c, d)$ to $R$ (since $(a, b)$ points to $(c, d)$ ). It then searches through the range $[c, d]$ and finalises the search (since block $(c, d)$ does not point to any other blocks). The fourth argument, mode, may be used to constrain the generation of the set $R$ and reduce the amount of reported information for the benefit of speed of execution. We further discuss the application of mode and describe different modes of operation of our technique in Subsection 3.1. For each element $(a, b) \in R$ the function update $\left(T_{\sigma}, a, l\right)$ (where $l$ is the input label) is executed which updates the usage labels associated with blocks $v$ references to $l$. The function $\operatorname{report}\left(T_{\sigma}\right)$ reports memory leaks based on the state of $T_{\sigma}$, such that for each element $\left(a, b, l_{a}, l_{u}\right)$ that belongs to $T_{\sigma}$, a memory block $(a, b)$ is reported as a memory leak, such that $(a, b)$ was allocated at location $l_{a}$ and last referenced by a variable at location $l_{u}$.

\subsection{Syntactic Transformations}

We now present the set of syntactic transformations, i.e., instrumenting the source code, in Figure 3. The first step of our transformation instruments an 
input program with a data structure $T_{\sigma}$ to keep track of the memory state (Figure 3, Rule Program).

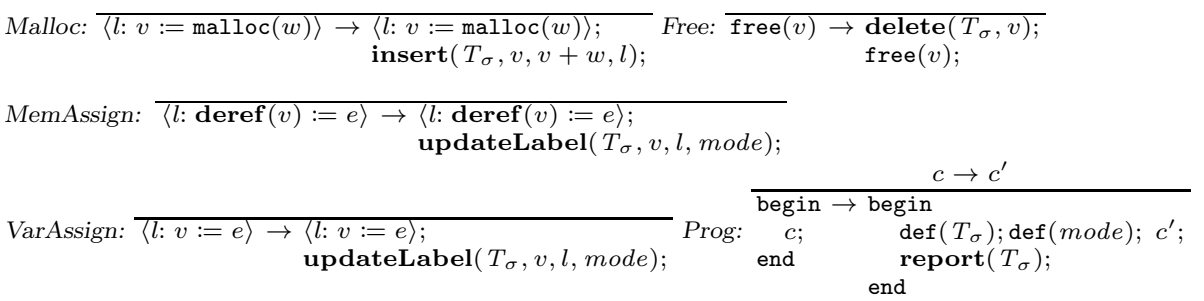

Fig. 3. Syntactic Transformations

Calls to insert and delete, which track allocated and de-allocated memory blocks, are injected into $P$ via rules Malloc and Free (Figure 3). That is, each statement that allocates memory (i.e., $\langle l: v:=\operatorname{malloc}(w)\rangle$ ) is followed by a call to $\operatorname{insert}\left(T_{\sigma}, v, v+w, l\right)$, where $v$ evaluates to the start address of the allocated block, expression $v+w$ evaluates to its end address and $l$ is a block's allocation label. Similarly, before each statement that de-allocates memory (i.e., a call to free $(\mathrm{v}))$, a call to $\operatorname{delete}\left(T_{\sigma}, v\right)$, that removes an element of $T_{\sigma}$ that refers to a de-allocated block, is made.

Each assignment statement is appended with a call to updateLabel, which tracks assignments of memory blocks referenced by variables. That is, calls to updateLabel update usage labels, which allows us to collect information on the propagation of data blocks. At any given state, a usage label associated with a block indicates a source location at which that block was last known to be referenced by a variable.

Since updateLabel is the main cause of the run-time overhead, its behaviour can be controlled externally by constraining the search for memory blocks referenced by a variable via limiting the search to the traversal of blocks of particular sizes only, where the maximal size of a traversed block is given by parameter mode. Currently, our approach supports Minimal, Partial and Full modes. In the Minimal mode updateLabel does not track usage labels. Hence, the reported information is limited to the existence of memory leaks and the locations of their allocation. In the Full mode, each block is traversed. In the Partial mode only the blocks of size less than mode are traversed. This avoids traversal of large blocks that may not contain pointers.

Finally, we insert a call to $\operatorname{report}\left(T_{\sigma}\right)$, before $P$ terminates.

At the dynamic stage of our approach we execute an instrumented program $P^{\prime}$, which reports memory leaks at the end of execution. A program run, for which $T_{\sigma}$ is empty, does not leak any memory. Otherwise, each element of $T_{\sigma}$ (say $\left.\left(a, b, l_{a}, l_{u}\right)\right)$ is reported as the memory leak of size $(b-a)$ allocated at program location $l_{a}$ and last known to be referenced by a variable at $l_{u}$. 


\subsection{Application on C Programs}

The technical details of the approach presented above are at the abstract level and need to be mapped to a concrete level to be able to apply them on a realistic programming language. We now discuss the extensions required to use our approach with $\mathrm{C}$ programs.

Due to the semantics of the $\mathrm{C}$ programming language, where stack-allocated memory blocks are automatically freed, additionally to a block's start and end addresses we record its type of allocation (i.e., stack, heap, global). This is to be able to distinguish between memory that is de-allocated explicitly or implicitly. Stack memory blocks are recorded to $T_{\sigma}$ explicitly, via inserting calls to insert immediately after definitions of local variables. The sizes of stack blocks are determined via the sizeof operator. Further, each stack block added to $T_{\sigma}$ is assigned an identifier of a scope it was allocated in.

Unlike in the abstract language, $\mathrm{C}$ statements are not labelled. To be able to generate all the required information we instrument the program with a stack that keeps track of entered functions and program locations associated with them. Thus the top element of the stack holds the location of the executed line, while other elements indicate locations of entered functions that lead to current function.

To be able to track all allocated heap memory we redefine memory allocation and de-allocation functions, which, apart from the normal functionality, insert or remove elements of $T_{\sigma}$. This is possible as the GNU C library defines malloc and similar functions as weak aliases. The original definitions of functions such as malloc are replaced with our instrumented ones. Such an approach allows for recording all heap memory, including blocks allocated by library functions for which no source code is available, e.g., strdup. Thus in practice we can report leaks for a larger class of programs than described formally.

Since in C programs values of pointers may be affected by function calls, function arguments are processed similarly to assignment statements.

\section{Results}

We have implemented our technique in a prototype tool called Skiff for programs written in the C programming language. Skiff is built on top of the Clang [6] compiler architecture (LLVM project, version 2.9 [7]). The platform for all results reported here was an Intel Core i5-2400 $3.1 \mathrm{GHz}$ machine with 4GB of RAM, running Gentoo Linux.

To evaluate the efficiency of our approach we have performed a number of experiments that involved instrumentation and dynamic analysis of well-known UNIX utilities, such as find, grep, gzip, diff, patch, rcs, locate and rm, and C benchmarks from the CPU2000 and CPU2006 sets developed by the Standard Performance Evaluation Corporation (SPEC). In this section we also report the results produced by Valgrind [8] (a state-of-the-art system for debugging and profiling programs) on the same test subjects and compare them to the results collected using our approach. 
During experimentation with UNIX utilities we monitored execution of their test suites and calculated overheads per test suite. Runs of CPU benchmarks were performed using the test data set provided by SPEC.

Note, that this evaluation focusses on the value of extended memory leak reports of the Full mode of Skiff and performance overheads, rather that on the number of discovered defects. This is because both techniques are sound and do not report false alarms. The reliability of Valgrind has been established by various experiments over the years. We manually checked that the output from Skiff is consistent with Valgrind's output.

We now report the results of our experimentation. We first outline differences in reporting of Full mode of Skiff and Valgrind and point out the benefits of locating sources of memory leaks. We then compare and discuss performance overheads incurred by different modes of Skiff and Valgrind.

Figure 4 demonstrates the difference in reporting schemes of Valgrind and Skiff in the Full mode. This uses a memory leak found in GNU locate (Findutils 4.4.2). The Valgrind report (on the left) shows the allocation site of this leak using a stack trace. Skiff (on the right) also uses stack traces to report leaks and shows the allocation site of the leak (a stack trace above the line of asterisks) and the source of leaked memory (a stack trace of below the line). Additionally, our tool reports variable names (e.g., procdata, highlighted in gray), that referenced the leaked memory block prior to the leakage. This removes ambiguity, as a single line of code in $\mathrm{C}$ may contain multiple statements.

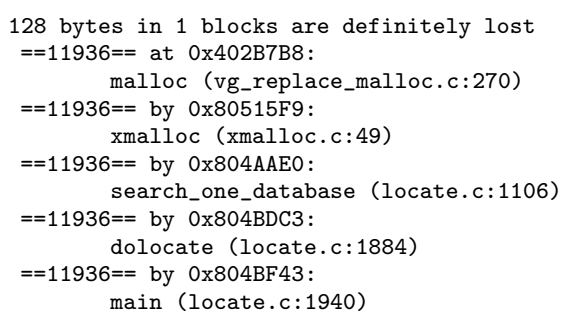

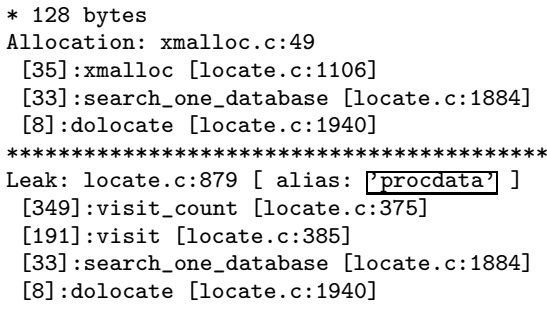

Fig. 4. Valgrind vs. Full Mode Memory Leak Report

We now discuss performance overheads of Skiff and Valgrind.

Figures [5] and [6] outline the difference in memory and run-time overheads produced by Valgrind and Skiff run in the Minimal mode. That is, the reports produced by both tools are similar and include detected memory leaks and their allocation sites as call traces. The Y-axis measures overhead ratio (comparing to the run-time or memory consumption of unmodified programs) and each point on the $\mathrm{X}$-axis stands for a series of runs of a program.

It can be seen that the run-time and memory overheads produced by our tool are lower than Valgrind's. The memory overhead produced by Skiff averages to $15 \%$ with the highest spike of approximately 3 times in equake only. Memory overheads of Valgrind are much higher, ranging from 1.6 to 34 times with the 


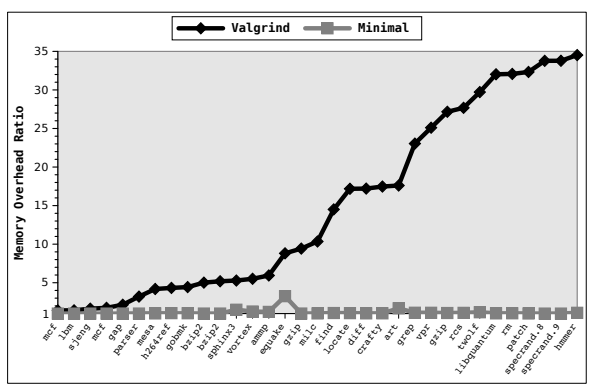

Fig. 5. Valgrind vs. Minimal Mode. Memory Overhead.

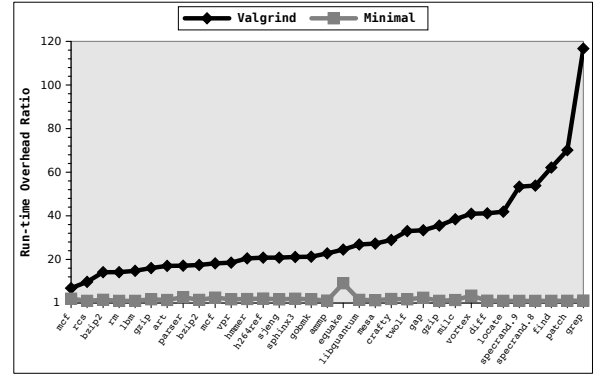

Fig. 6. Valgrind vs. Minimal Mode. Run-time Overhead.

average of 15 times. The run-time overhead exhibited by both tools compares similarly. The overhead produced by Skiff is on average approximately $80 \%$, while the average run-time overhead of Valgrind is 30.8 times, ranging from 6.8 to 116 times. Note, that spikes, such as 116 times in grep can be partially attributed to a high number of invocations of programs during test suite execution (e.g., over 1250 runs in grep test suite), where each invocation causes Valgrind to dynamically instrument a binary monitor memory. This is different to our approach, where we eliminate such overhead by instrumenting source code and compile monitoring capabilities in. Additionally, we track memory at the block level and store only the delta of information, such as block addresses, sizes etc., whereas Valgrind monitors each byte individually. That is, our overhead is proportional to the number of memory blocks allocated by a program, whereas Valgrind's is proportional to the overall amount of allocated memory. Note, that while on average block-level tracking proves to yield low overhead, it may increase if many small blocks are allocated. This is exhibited, by the equake benchmark where overheads can be 3 times for memory and 9 times for run-time. Finally, it should be noted that in the Minimal mode we monitor only allocation and de-allocation operations, which is computationally light. Of course, this does not produce useful debugging information.

The run-time overhead in the Full mode varies and may increase significantly based on sizes of memory blocks a program manipulates. This is because our main run-time overhead is due to computation, i.e., iteration through address ranges of memory blocks and identifying pointers in assignments. Thus, the main factor that influences overhead is the size of memory blocks traversed (i.e., size of manipulated data structures) and the frequency of their use (i.e., the number of statements that trigger updateLabel). Thus, we may expect larger overheads for SPEC benchmarks, as these programs are crafted to routinely perform computationally intensive tasks (such as archiving, compilation etc.) on large data chunks. We now discuss the results of our experimentation.

Figure 7 depicts the run-time overheads of our prototype run in the Full and Minimal modes and Valgrind on the set of UNIX utilities. It can be seen that in the Full mode our overhead increases, spanning from 1.3 times (in gzip) to 
almost 11 times in the rcs test suite. These overheads, however, are still lower than the overheads produced by Valgrind. Notably, the memory overhead does not increase significantly, reaching the maximum of $21 \%$ in UNIX programs. From Figure [5] it can be seen that this is approximately 15 to 30 times the overheads incurred by Skiff. Including the results from Valgrind in one graph obscures the difference in performance between the two modes.

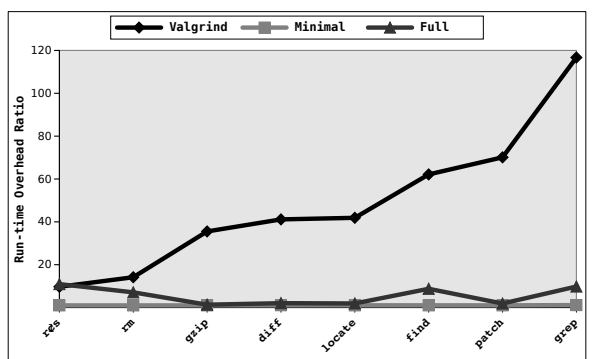

Fig. 7. UNIX: Run-time Overhead

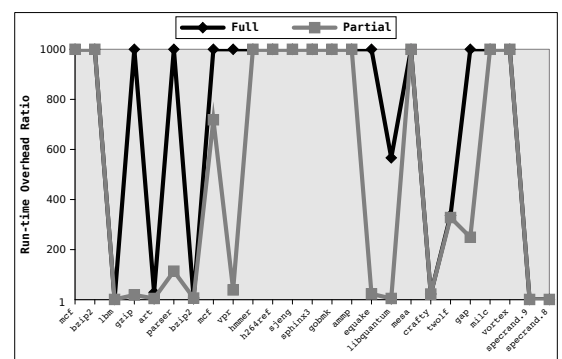

Fig. 8. SPEC CPU: Run-time Overhead

It is important to note that Skiff in Full mode does not always outperform Valgrind - especially on the SPEC CPU benchmarks. Sometimes our run-time overhead is extremely high (over 1000 times). The main factor that contributes to such overhead is the size of memory blocks allocated. The larger the size of the memory allocated, the longer it takes to run updateLabel. This behaviour is confirmed via experimentation in Partial mode. When we limit traversal of memory blocks by the size of the largest data structure, i.e., assuming that larger blocks are data only blocks and do not contain any pointers, the overheads are significantly reduced as depicted in Figure 8 which for the sake of clarity does not show the data associated with Valgrind. One can compare the performance of Valgrind and Skiff in Full mode by combining the data from Figures 6 and 8 The raw data is available from the authors on request.

For example, the overhead for the $1 \mathrm{bm}$ benchmark is reduced from 566 to only 5 times. The excessive overhead of benchmarks, which continue to perform similarly to the Full mode, is due to the structure of some SPEC benchmarks, where a large amount of memory is allocated statically, irrelevant of the input size. Note, that while developing programs such allocations are rarely used.

The relation between increased run-time overhead and the amount of memory allocated by programs is illustrated in Figure 9. The presentation of these data is split because of the difference in the run-time overhead ratios and thus the scale of the figures is different. In the set of UNIX utilities (Figure 9, leftmost plot) the main purpose of the associated test suites is to evaluate functional correctness of programs, where memory consumption does not exceed 5 megabytes and thus Skiff, even in Full mode, outperforms Valgrind. In the SPEC CPU benchmarks (Figure 9, center and rightmost plots) memory consumption is much higher. The center plot of Figure 9 shows that Skiff in Minimal mode always outperforms 

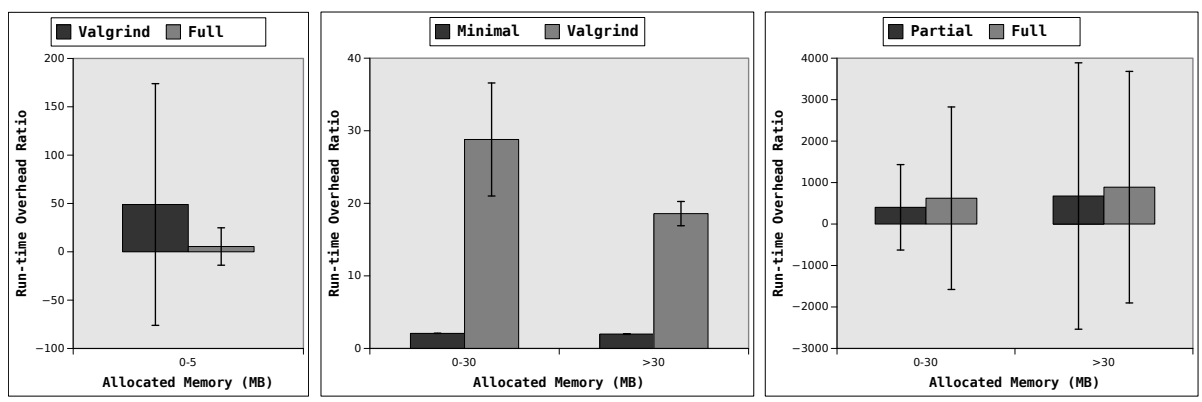

Fig. 9. Overhead Relative to Memory Usage. Left to right: UNIX Programs (Full vs. Valgrind), SPEC CPU (Minimal vs. Valgrind), SPEC CPU (Partial vs. Full).

Valgrind with negligible overheads and low variance. The rightmost plot of Figure 9 compares the overheads incurred by Skiff in Partial and Full modes. Note, memory size alone does not affect the overheads. If each allocated block contains pointers, then these pointers need to be tracked, adding to overheads, while if the allocated blocks contain only data they need not be tracked, reducing the overheads. This is shown by the variance in the overheads. What this means is that the overhead is not consistently high. Note that abnormal cases with extreme overhead in SPEC CPU should be attributed to the design pattern of SPEC benchmarks which are aimed at performance evaluation. The memory consumption, however, affects only Full or Partial modes.

Our approach is mainly useful in the domain of functional testing, where program correctness is established through runs with small inputs. In addition to memory leak detection, our technique can provide useful information that facilitates debugging. Note, that our experimentation with UNIX utilities indicates that with small inputs Skiff overheads may be lower than the overheads of conventional monitoring using Valgrind. Our approach can also be used in performance testing, where program runs are significantly heavier, in both memory consumption and run-time. Our experimentation suggests that for memory leak detection our technique uses considerably less resources than DBI, while still producing the same level of output, especially in Minimal or Partial mode.

\subsection{Threats to Validity}

We now discuss factors that may have affected the validity of our results. The first factor is the choice of programs and the input data used in our experimentation. Even though, during experimentation with UNIX utilities we used realistic programs and representative inputs (i.e., test suites that are associated with the utilities), which should account for exercising most of the paths, there is no evidence that applying our technique on different programs or using different input values will yield similar results. Similarly, during our second experiment with SPEC CPU benchmarks, the input values provided may not be representative for the development process. This is because SPEC 
concentrates on performance evaluation, rather that on exploring various behaviours. The second issue refers to our comparison with Valgrind. Valgrind is a memory profiler, whose core functionality goes far beyond just memory leak detection. Consequently, some overhead produced by Valgrind may be attributed to performing tasks not relevant for memory leak detection. But our tool is only a proof-of-concept implementation, while Valgrind is more robust. Thus, a better implementation of our technique may improve the results.

\section{Related Work}

The memory leak problem has been extensively researched, resulting in a variety of memory leak detection techniques that use static and dynamic analyses. We now summarise papers that are directly relevant to our approach.

One of the most common approaches in memory profiling is to track memory usage at the byte level via binary instrumentation. One of the earliest attempts is Purify [1. Purify statically inserts additional instructions directly into object files monitoring memory allocation and every read or write performed by a program under test. More recent approaches, such as Dr. Memory [9], use dynamic binary instrumentation (DBI). In DBI, an executable is analysed using extra code added to it at run-time. Despite the strengths of DBI, such as soundness and the ability to monitor any memory operation, DBI may produce high overhead in both execution time and memory consumption, while still reporting only allocation sites of leaked blocks. In our approach we have the benefit of locating the source of leakage without incurring the overheads associated with DBI.

Insure++ [3] is a memory profiler for $\mathrm{C}$ and $\mathrm{C}++$. Unlike profilers that exclusively instrument binaries, Insure++ can operate at the source code level. Unfortunately, implementation details of this tools is not publicly available.

Determining precise locations of memory leakage has also been investigated. Maebe et al. [10] have presented a technique that tracks all pointers to the allocated memory using reference count. In their approach authors keep track of pointers by monitoring load and store operations that may change pointer structure of a program, detecting locations of memory leakage. The downside of this technique is that it can report both false positive and false negative results. Clause and Orso [11] developed a similar technique that detects sources of memory leaks, called LEAKPOINT. LEAKPOINT tracks memory using dynamic taint analysis. A tainted pointer identifies an access alias to that memory block. LEAKPOINT updates the taint as execution proceeds by observing operations on pointers. This uses a propagation policy which models each such operation. At run-time LEAKPOINT keeps track of pointer count per allocated memory block (i.e., taint marks associated with pointers) and identifies leakage locations as the locations where pointer count dropped to zero. The main weakness of LEAKPOINT is that its propagation policy is neither sound nor complete. That is, while LEAKPOINT may soundly identify memory leak existence, the reported sources of leaked memory are not guaranteed to be correct. Finally, LEAKPOINT is a DBI approach (built on top of Valgrind) and thus also suffers from very high 
overheads (e.g., the authors report overheads that are 300 times) Our technique addresses a similar question by using on-the-fly computation rather than reference count. This makes our technique both sound and complete which we have formally proven. Also note, that our technique correctly handles cases such as $\mathrm{p} 1=\mathrm{p} 2+1$; and $\mathrm{p}++$; where $\mathrm{p}$ is moved past the end of a block.

Another approach that can potentially detect location of leakage, is Boehm GC [12 - a garbage collector for $\mathrm{C}$ and $\mathrm{C}++$ languages that uses a variation of the mark-and-sweep algorithm. If used as a leak detector, Boehm GC reports memory blocks that are no longer accessible as memory leaks. This, however, relies on a periodic scanning of program address space to determine lost memory and thus can not determine precise locations of leakage.

\section{Conclusions}

We have presented a tunable monitoring approach to locating memory leaks. Our technique uses source-to-source transformations to instrument an input program with statements to monitor its memory state of and report leaks before the modified program terminates. Our approach has the benefit of locating program points at which leaked memory was lost. This aids the debugging process, providing developers with extra information that can be then used to eliminate detected defects. Additionally, our approach provides tuned monitoring via different modes of execution enabled at run-time. In the Full mode extra information of leakage locations is produced for the cost of larger overheads. Minimal mode minimises overheads using a conventional reporting scheme that outputs only allocation sites. Partial mode reduces overheads by tracking leakage locations of memory blocks of particular sizes only.

We have implemented our approach in a prototype tool called Skiff for C programs. During our experimentation we monitored execution of a number of well-known UNIX applications and SPEC CPU benchmarks. Further, the results of our tool were compared to the results produced by Valgrind. Our experimentation shows that in conventional reporting, where only allocation sites of leaked blocks are determined, our approach significantly outperforms Valgrind. Such results indicate that for memory leak detection Skiff may be used as a replacement for binary instrumentation tools, producing similar results with considerably less system resources. Experimentation with Full mode shows that our overheads directly depend on the amount of memory allocated by programs and increase as memory consumption grows. Analysis of UNIX utilities showed Skiff performed better than Valgrind mainly due to relatively small allocated blocks. However, Skiff performed considerably worse on SPEC benchmarks, which focus on performance evaluation and thus use large inputs. We demonstrated the applicability of overhead tuning using Partial mode, where in some cases we reduced large overheads of SPEC benchmarks by not tracking large data blocks for leakage. Overall we can conclude that leakage detection at the current stage is mainly useful in the domain of functional testing, where program correctness is established with runs with relatively small inputs. 
In the future we are looking to improving the performance of our technique for large allocations. Our preliminary experimentation shows that the high overheads incurred are due to tracking blocks that do not leak or are accessible globally. Thus, rather than instrumenting each statement, we could use lightweight but sound static program analysis to filter out statements that cannot leak. We would also like to consider alternate or extended instrumentations that detect other memory faults, such as, for example, use after free errors.

Acknowledgement. The first author is supported by a grant from Oracle Labs. The second author was affiliated with Bond University when most of this work was done.

\section{References}

1. Hastings, R., Joyce, B.: Purify: Fast detection of memory leaks and access errors. In: Proceedings of the Winter USENIX Conference, pp. 125-136 (January 1992)

2. Seward, J., Nethercote, N.: Using valgrind to detect undefined value errors with bitprecision. In: Proceedings of the USENIX Annual Technical Conference, USENIX, pp. 17-30 (2005)

3. Parasoft (Insure++), http://www.parasoft.com/jsp/products/insure.jsp

4. Intel: (Parallel inspector), http://software.intel.com/en-us/intel-parallel-inspector

5. Nielson, H.R., Nielson, F.: Semantics with applications - a formal introduction. Wiley Professional Computing. Wiley (1992)

6. clang: a C language family frontend for LLVM (March 2012), http://clang.llvm.org

7. Lattner, C., Adve, V.: LLVM: A Compilation Framework for Lifelong Program Analysis \& Transformation. In: Proceedings of the International Symposium on Code Generation and Optimization, CGO 2004. IEEE Computer Society, Washington, DC (2004)

8. Nethercote, N., Seward, J.: Valgrind: A framework for heavyweight dynamic binary instrumentation. In: Proceedings of the ACM SIGPLAN Conference on Programming Language Design and Implementation, PLDI 2007, vol. 42, pp. 89-100. ACM, New York (2007)

9. Bruening, D., Zhao, Q.: Practical memory checking with Dr. Memory. In: Proceedings of the Annual IEEE/ACM International Symposium on Code Generation and Optimization, CGO 2011, pp. 213-223. IEEE Computer Society, Washington, DC (2011)

10. Maebe, J., Ronsse, M., Bosschere, K.D.: Precise detection of memory leaks. In: Proceedings of the International Workshop on Dynamic Analysis, pp. 25-31 (May 2004)

11. Clause, J.A., Orso, A.: LEAKPOINT: Pinpointing the causes of memory leaks. In: Proceedings of the ACM/IEEE International Conference on Software Engineering, ICSE 2010, vol. 1, pp. 515-524. ACM (May 2010)

12. Boehm, H.: Dynamic memory allocation and garbage collection. Computers in Physics 9, 297-303 (1995) 